\title{
Double surface imaging designs with unconstrained object to image mapping under rotational symmetry
}

\author{
Jiayao Liu*a, Juan C. Miñano $^{\mathrm{ab}}$, Pablo Benítez ${ }^{\mathrm{ab}}$ \\ ${ }^{a}$ Universidad Politécnica de Madrid, Cedint, Campus Montegancedo, Pozuelo, 28223 Madrid, Spain; \\ ${ }^{\mathrm{b}} \mathrm{LPI}, 2400$ Lincoln Avenue, Altadena, CA 91001, USA
}

\begin{abstract}
In this work, we present a novel imaging design formed by two optical surfaces with rotational symmetry. In these designs, both object and image shapes are given but mapping from object to image is obtained through the design process. In the examples considered, the image from a planar object surface is virtual and located at infinity and is seen from a known pupil, which can emulate a human eye.

The differential equation method is used to provide single optical surface imaging designs by considering the local properties of the imaging surface and the wavefronts. In the first introductory part, both the rotational symmetrical and the freeform single surface imaging designs are presented using the differential equation method. In these designs, not only the mapping is obtained in the design process, but also the shape of the object is found.

In the second part, the method is extended to two surface designs with rotational symmetry and the astigmatism of the image has been studied. By adding one more optical surface to the system, the shape of the rotational symmetrical object can be designed while controlling the tangential rays and sagittal rays simultaneously. As a result, designs without astigmatism (at the small pupil limit) on a planar object surface have been obtained.
\end{abstract}

Keywords: Head-mounted displays, imaging, anastigmatic, differential geometry

\section{INTRODUCTION}

The first graphics-driven head-mounted display (HMD) was developed by Ivan Sutherland in the 1960s ${ }^{1}$. This display system is supposed to be worn on the human head, providing three-dimensional interactive displays while not limiting not only the free motion of the head, but also the potential body movement. Considering the fact that the humans have not evolved to wear heavy devices on the head, the lightweight and ergonomics have been the important criteria for the design of $\mathrm{HMD}^{2}$.

A simple optical system, like a single reflective system or a single lens system with double surfaces, can help to fulfill the design criteria. Aspheric and freeform optics are required to provide good performance of the optical system. There are various approaches to generate aspheric and freeform optic profiles: Simultaneous Multiple Surface (SMS) method ${ }^{3,4}$, differential equations based on surface slopes ${ }^{5}$, analytical solutions to surface profiles ${ }^{6,7}$, etc. These aspheric and freeform optics can be fitted with a series of representation forms ${ }^{8,9}$ for potential optimization or manufacturing process.

We have presented a differential equation based single surface imaging design approach in previous papers ${ }^{10,11}$. This approach considers both the tangential and sagittal ray propagation and can readily provide anastigmatic optical surface profile. The image surface and the mapping from the object to the image are obtained after the design. The anastigmatic design obtained acts as a good starting point for the final solution after subsequent optimization.

In this paper, we extend the former single surface optical design approach to a double surface optical design approach. The one more optical surface added can provide additional freedom to the design. The shape of the image surface can be controlled with this additional freedom. Thus, a double surface image design with a flat image surface is obtained. The mapping from the object to the image is obtained after the design.

\section{SINGLE SURFACE DESIGNS}

The design approach presented in this section is for 3D rotational designs and 3D freeform designs. Due to the reversibility of optical path, the design can be considered as a design of a single optical surface forming a mapping of the 
rays from the source, which is the pupil, with angle $\theta$ respected to the optical axis to the image plane, which is the object, at the point $x(\theta)$. The sketch map of the refractive and reflective design is shown in Fig. 1.

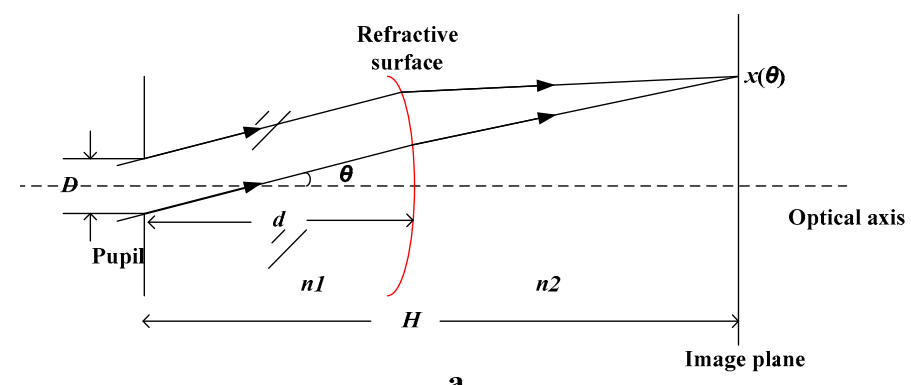

a

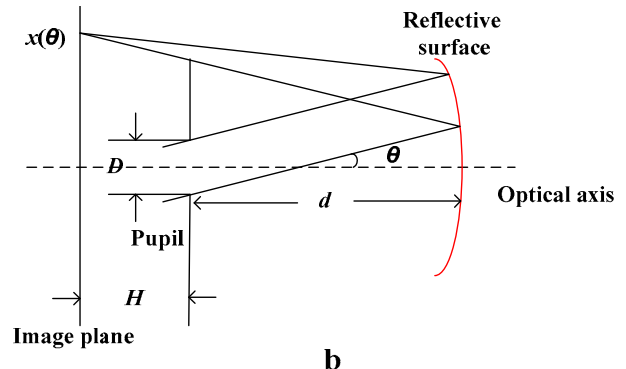

Figure 1. Sketch map of the refractive design (a) and the reflective design (b)

The generalized ray tracing can be expressed by local properties of the surface and wavefronts such as slopes and curvatures by the following equations known as Coddington equations ${ }^{12}$ :

$$
\left\{\begin{aligned}
\frac{n_{o} \cos ^{2} \theta_{o}}{\rho_{q o}} & =\frac{n_{i} \cos ^{2} \theta_{i}}{\rho_{q i}}+\frac{n_{o} \cos \theta_{o}-n_{i} \cos \theta_{i}}{\rho_{q s}} \\
\frac{n_{o}}{\rho_{p o}} & =\frac{n_{i}}{\rho_{p i}}+\frac{n_{o} \cos \theta_{o}-n_{i} \cos \theta_{i}}{\rho_{p s}}
\end{aligned}\right.
$$

Where $n$ denotes the refractive index, $\rho$ denotes the radius of curvature and $\theta$ denotes the angle between the ray and the normal of the surface. Subscripts $i$, $o$ and $s$ refer to input, output and surface. Subscripts $q$ and $p$ refer to the plane of incidence and the one rotated 90 degrees about the incident ray respectively.

\subsection{D rotational designs}

Considering an infinitesimal part in polar coordinate $(r(\alpha), \alpha)$, we can derive the slope and the radius of curvature in the plane of incidence in differential form as follows:

$$
\theta=\theta\left(r, \alpha, \frac{d r}{d \alpha}\right), \rho_{q}=\rho_{q}\left(r, \alpha, \frac{d r}{d \alpha}, \frac{d^{2} r}{d \alpha^{2}}\right) .
$$

For designs with rotational symmetry, the $q$ and $p$ directions in Eq. 1 are tangential and sagittal directions and the radius of curvature in the rotated plane about the incident ray is the function of the slope in the plane of incidence, thus can be expressed as Eq. 3.

$$
\rho_{p}=\rho_{p}\left(r, \alpha, \frac{d r}{d \alpha}\right)
$$

Combining the above formulations, we can derive a second-order ordinary differential equation. The numerical solution from specific boundary condition represents a specific 3D rotational optical surface profile. We have derived anastigmatic designs for plane input wavefront shown in Fig. 2. 

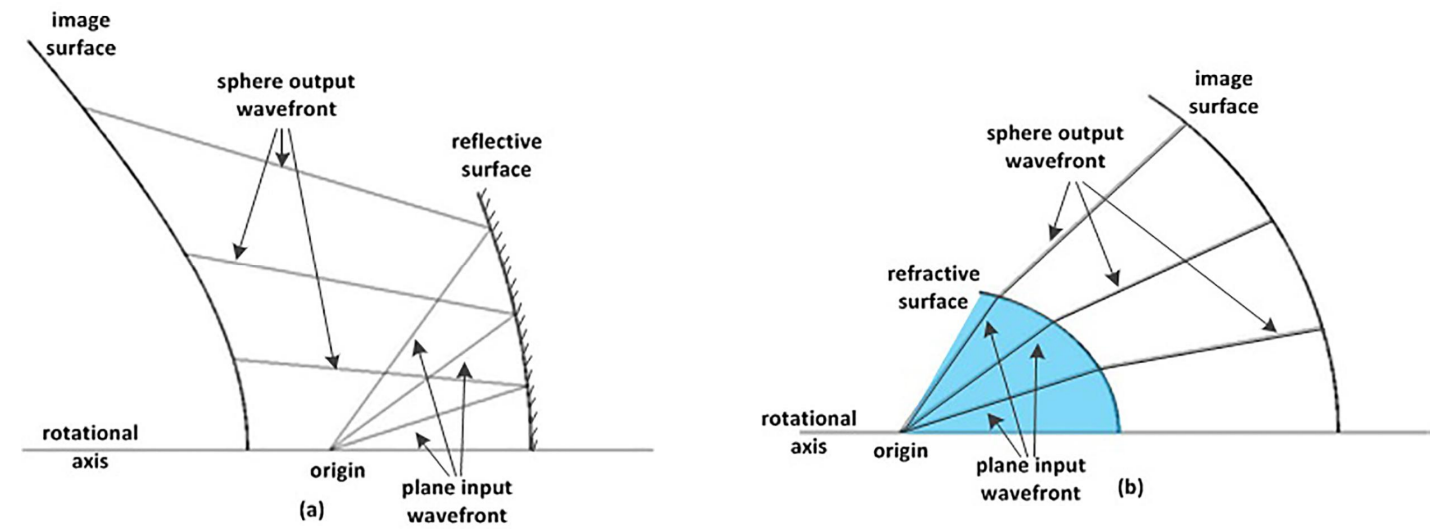

Figure 2. The reflective anastigmatic rotational design (a) and the refractive anastigmatic rotational design (b). The image surface profile is calculated after the design of the optical surface

The image surface and the mapping from object to image are obtained from the optical surface profile.

\subsection{D freeform designs}

For 3D designs without rotational symmetry in spherical coordinates $(r(\alpha, \beta), \alpha, \beta)$, the curvature in the rotated plane about the incident ray is no longer the function of the slope in the plane of incidence.

In differential geometry, the two principal radii of curvature can be given as the two solutions of the following equation ${ }^{13}$ :

$$
\left(E G-F^{2}\right) \rho^{2}-(E N+G L-2 F M) \rho+L N-M^{2}=0
$$

In this equation, $E, F, G$ are the first fundamental quantities which are functions of $r, \alpha, \beta, \partial r / \partial \alpha$ and $\partial r / \partial \beta$ and $L, N, M$ the second fundamental quantities which are functions of $\partial^{2} r / \partial \alpha^{2}, \partial^{2} r / \partial \beta^{2}$ and $\partial r^{2} / \partial \alpha \partial \beta$ besides $r, \alpha, \beta, \partial r / \partial \alpha$ and $\partial r / \partial \beta$. We can derive a partial differential equation by substituting the two solutions into Eq. 1. Similar to rotational cases, the numerical solution from specific boundary condition represents specific 3D freeform optical surface profile.

For designs with plane input wavefront and spherical output wavefront condition, we can derive a second order hyperbolic partial differential equation.

$$
a \frac{\partial^{2} r}{\partial \alpha^{2}}+b \frac{\partial^{2} r}{\partial \alpha \partial \beta}+c \frac{\partial^{2} r}{\partial \beta^{2}}=e
$$

We have derived a 3D freeform reflective anastigmatic design shown in Fig. 3. The boundary condition is chosen that the initial curve of the image surface is a straight line, so that the image surface obtained after the design is much closer to a flat plane, which is more applicable in real applications. The mapping from object to image is obtained from the optical surface profile.

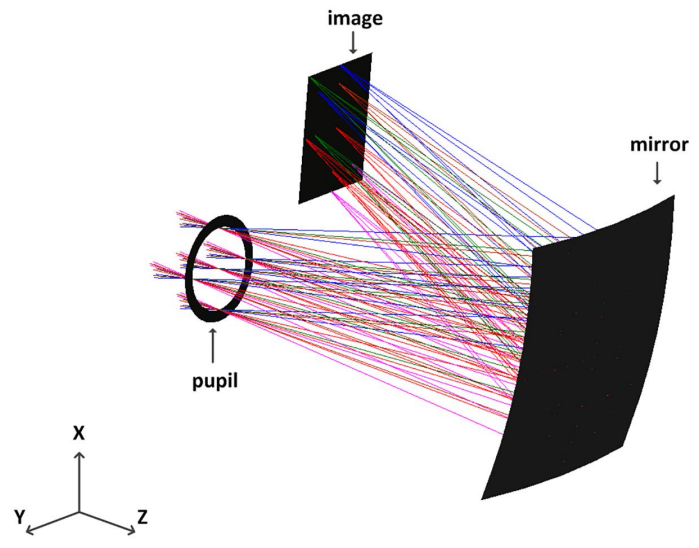

Figure 3. 3D freeform reflective anastigmatic design

This approach is also applicable for refractive designs. 


\section{DOUBLE SURFACE DESIGN}

The differential equation presented in section 2 removes the freedom from controlling the mapping from the object to the image, and uses this freedom to control the sagittal ray propagation. But the single optical surface is incapable of providing additional freedom to control the mapping or the shape of the image surface. Even in 3D freeform designs in section 2 , the appropriate choice of the boundary condition can only provide an image surface closer to a flat one (Fig, 4).

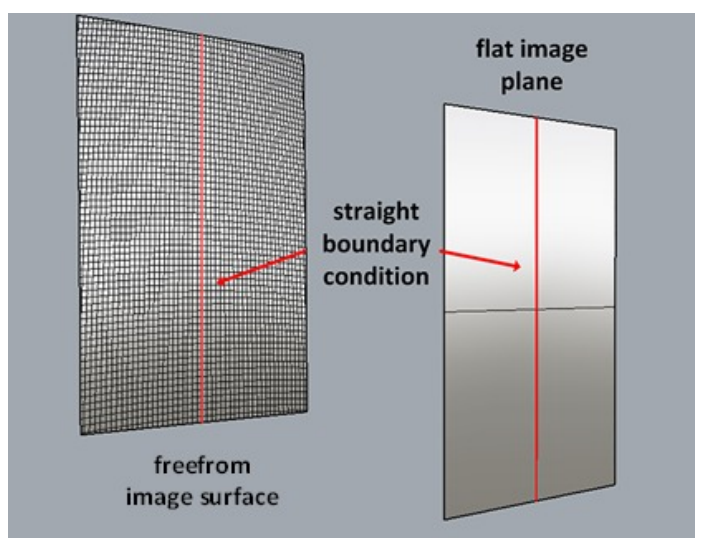

Figure 4. The flat image plane and the freeform image surface

In some cases, more freedom is required to control the mapping or the shape of the image surface. Thus we have extended the former differential equation method to the double surface design with rotational symmetry.

We consider the refractive double surface optical system with rotational symmetry which has a flat image plane as shown in Fig. 5.

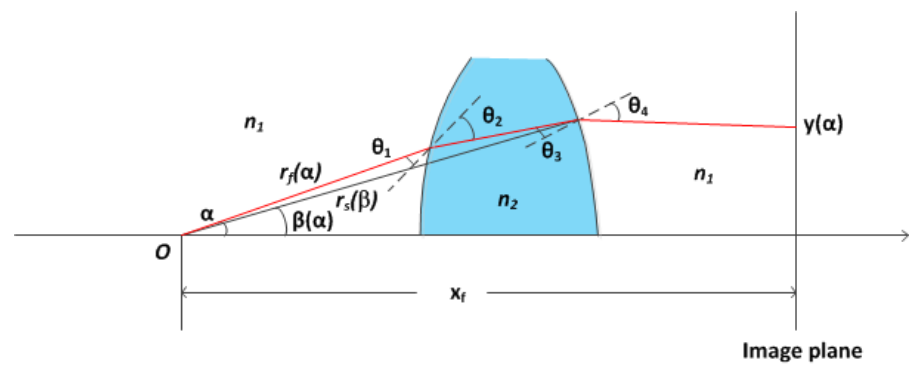

Figure 5. Sketch map of the double surface optical system with flat image plane

The first and the second optical surfaces are expressed by $\left(r_{\mathrm{f}}(\alpha), \alpha\right)$ and $\left(r_{\mathrm{s}}(\beta), \beta\right)$ respectively.

The Eq. 1 describes the ray propagation in both the first surface and the second surface as shown in Eq. 6 :

$$
\left\{\begin{array}{rl}
\frac{n_{2} \cos ^{2} \theta_{2}}{\rho_{2 q}} & =\frac{n_{1} \cos ^{2} \theta_{1}}{\rho_{1 q}}+\frac{n_{2} \cos \theta_{2}-n_{1} \cos \theta_{1}}{\rho_{f q}} \\
\frac{n_{2}}{\rho_{2 p}} & =\frac{n_{1}}{\rho_{1 p}}+\frac{n_{2} \cos \theta_{2}-n_{1} \cos \theta_{1}}{\rho_{f p}}
\end{array},\left\{\begin{array}{c}
\frac{n_{1} \cos ^{2} \theta_{4}}{\rho_{4 q}}=\frac{n_{2} \cos ^{2} \theta_{3}}{\rho_{3 q}}+\frac{n_{1} \cos \theta_{4}-n_{2} \cos \theta_{3}}{\rho_{s q}} \\
\frac{n_{1}}{\rho_{4 p}}=\frac{n_{2}}{\rho_{3 p}}+\frac{n_{1} \cos \theta_{4}-n_{2} \cos \theta_{3}}{\rho_{s p}}
\end{array}\right.\right.
$$

Where 2 and 3 refer to the wavefronts after the first surface and the wavefronts before the second surface respectively.

Between the two surfaces, the transformation of the radii of curvature of the wavefronts are shown in Eq. 7 :

$$
\rho_{2 q}-D=\rho_{3 q}, \rho_{2 p}-D=\rho_{3 p}
$$

The parameter $D$ is the distance between the two points on the two surfaces where the refraction occurs.

The Eq. 7 unifies the two pairs of equations in Eq. 6 describing the ray propagation on the first and the second surface. Thus we can derive equations to describe the ray propagation for the entire system (Eq. 8). 


$$
\left\{\begin{array}{l}
\frac{n_{2} \cos ^{2} \theta_{3}}{\frac{n_{2} \cos ^{2} \theta_{2} \cdot \rho_{f q}}{n_{2} \cos \theta_{2}-n_{1} \cos \theta_{1}}-D}=\frac{n_{1} \cos ^{2} \theta_{4}}{\rho_{4 q}}-\frac{n_{1} \cos \theta_{4}-n_{2} \cos \theta_{3}}{\rho_{s q}} \\
\frac{n_{2}}{\frac{n_{2} \rho_{f p}}{n_{2} \cos \theta_{2}-n_{1} \cos \theta_{1}}-D}=\frac{n_{1}}{\rho_{4 p}}-\frac{n_{1} \cos \theta_{4}-n_{2} \cos \theta_{3}}{\rho_{s p}}
\end{array}\right.
$$

For anastigmatic designs, the output wavefronts are spherical, i.e., $\rho_{4 \mathrm{q}}=\rho_{4 \mathrm{p}}$ in Eq. 8 . This condition can be expressed by the following equation:

$$
\frac{n_{1} \cos ^{2} \theta_{4}}{\frac{n_{2} \cos ^{2} \theta_{3}}{\frac{n_{2} \cos ^{2} \theta_{2} \cdot \rho_{f q}}{n_{2} \cos \theta_{2}-n_{1} \cos \theta_{1}}-D}+\frac{n_{1} \cos \theta_{4}-n_{2} \cos \theta_{3}}{\rho_{s q}}}=\frac{n_{1}}{\frac{n_{2}}{\frac{n_{2} \rho_{f p}}{n_{2} \cos \theta_{2}-n_{1} \cos \theta_{1}}-D}+\frac{n_{1} \cos \theta_{4}-n_{2} \cos \theta_{3}}{\rho_{s p}}}
$$

The flat image plane condition gives the values of the radii of curvature of the outgoing spherical wavefronts.

$$
\rho_{4 q}=\rho_{4 p}=\frac{x_{f}-r_{s} \cos \beta}{\cos \left(\beta-\arctan \left(\frac{d r_{s}}{d \beta}\right)+\theta_{4}\right)}
$$

The two optical surfaces are connected by the law of sines:

$$
\frac{r_{s}}{\sin \left(\pi-\left(\theta_{1}-\theta_{2}\right)\right)}=\frac{r_{f}}{\sin \left(\beta-\alpha+\theta_{1}-\theta_{2}\right)}
$$

In Eq. 9, 10 and 11:

$$
\begin{array}{cc}
\theta_{1}=\theta_{1}\left(r_{f}, \alpha, \frac{d r_{f}}{d \alpha}\right) & \theta_{3}=\theta_{3}\left(r_{f}, \alpha, \frac{d r_{f}}{d \alpha}, r_{s}, \beta, \frac{d r_{s}}{d \beta}\right) \\
\rho_{f q}=\rho_{f q}\left(r_{f}, \alpha, \frac{d r_{f}}{d \alpha}, \frac{d^{2} r_{f}}{d \alpha^{2}}\right) & \rho_{f p}=\rho_{f p}\left(r_{f}, \alpha, \frac{d r_{f}}{d \alpha}\right) \\
\rho_{s q}=\rho_{s q}\left(r_{s}, \beta, \frac{d r_{s}}{d \beta}, \frac{d^{2} r_{s}}{d \beta^{2}}\right) & \rho_{s p}=\rho_{s p}\left(r_{s}, \beta, \frac{d r_{s}}{d \beta}\right)
\end{array}
$$

$\theta_{2}$ and $\theta_{4}$ can be derived by $\theta_{1}$ and $\theta_{3}$ using Snell's law.

Substituting Eq. 11 into Eq. 9 and Eq. 10 and applying the expressions shown in Eq. 12, we can derive two implicit second order ordinary differential equations with dependent variables $r_{f}$ and $r_{s}$ in functions of one single independent variable $\alpha$. The numerical solution from specific boundary condition represents specific 3D rotational optical surface profiles.

\section{DOUBLE SURFACE DESIGN EXAMPLE}

We have derived a refractive anastigmatic design with a flat image plane using the approach presented in section 3 . The input rays come from the origin with input angle $\alpha$ respect to the optical axis. For every input angle, the input wavefront 
is a plane wavefront, thus the parameters $\rho_{1 \mathrm{q}}=\rho_{\mathrm{lp}}=\infty$. The vertex of the first optical surface is on the optical axis and is 3.15 from the origin; the vertex of the second optical surface is also on the optical axis and is 4.15 from the origin; the image plane is perpendicular to the optical axis and is set to be 8.5 from the origin. All the dimensions are of arbitrary units. The material between the two surfaces is PMMA and the rest parts are vacuum with refractive index of 1 . The whole system is rotationally symmetrical about the optical axis.

The numerical solution of the implicit second order ODE from conditions listed above represents a lens profile shown in Fig. 6.

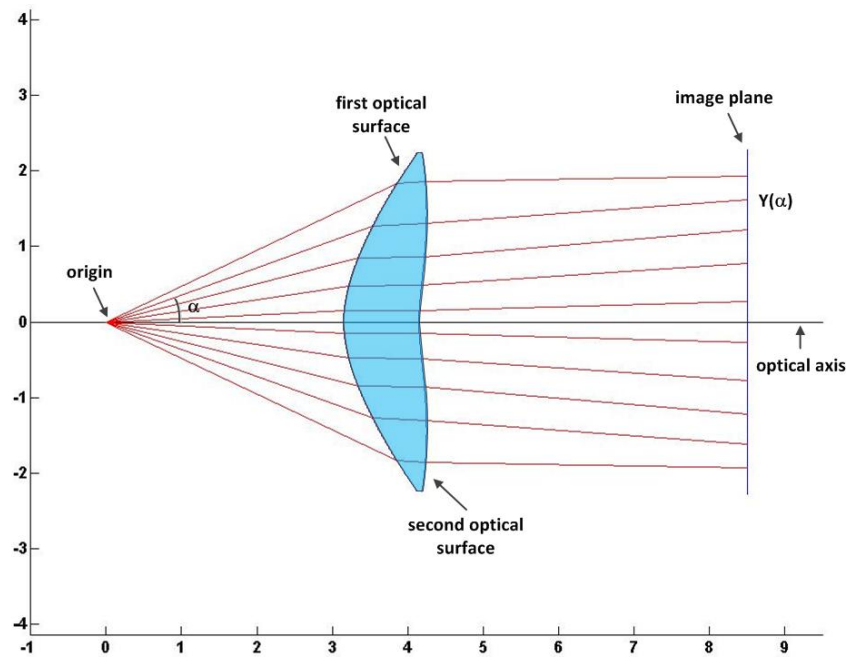

Figure. 6 The refractive double surface anastigmatic design with flat image plane

The tangential image plane and sagittal image plane are calculated separately from the lens profile. The difference between the two image planes is evaluated by the difference of the radii of curvature of the tangential and sagittal output wavefronts (Fig. 7).

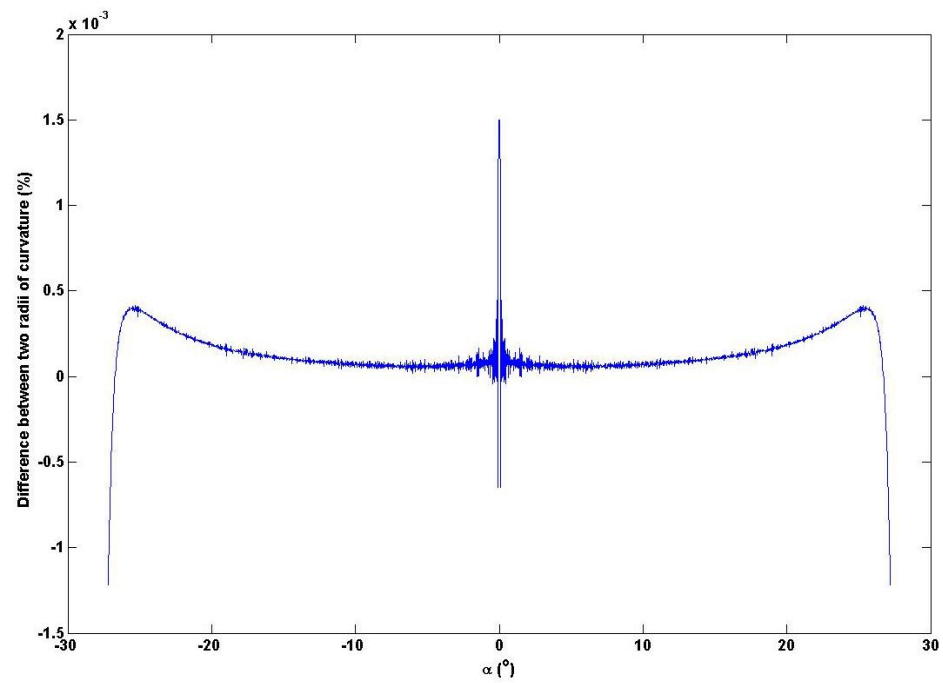

Figure. 7 Difference between two radii of curvature of the output wavefronts in percentage of the average value

The maximum difference is less than $0.002 \%$ of the average value, which indicates that the design is anastigmatic. The mapping $Y(\alpha)$ from the origin to the image is obtained from the lens profile.

\section{CONCLUSION}

The Coddington equations describe both the tangential and sagittal ray propagations. Based on these equations, the ODE and PDE design approaches provide the freedom to control the tangential and sagittal ray propagations simultaneously 
during optical designs. The single surface anastigmatic designs can be readily obtained but the mapping from object to image and the shape of the image surface is uncontrollable.

The single surface differential approach has been extended to double surface designs which provide more freedom to control the mapping or the shape of the image surface. Applying this extended approach, a refractive double surface design with flat image plane has been derived. The result of the verification of the design has indicated that the design is anastigmatic as the design goal. This approach is also applicable for reflective designs.

\section{ACKNOWLEDGMENTS}

Authors thank the European Commission (SMETHODS: FP7-ICT-2009-7 Grant Agreement No. 288526, NGCPV: FP7ENERGY.2011.1.1 Grant Agreement No. 283798), the Spanish Ministries (ENGINEERING METAMATERIALS: CSD2008-00066, SEM: TSI-020302-2010-65 SUPERRESOLUCION: TEC2011-24019, SIGMAMODULOS: IPT2011-1441-920000, PMEL: IPT-2011-1212-920000), and UPM (Q090935C59) for the support given to the research activity of the UPM-Optics Engineering Group, making the present work possible.

\section{REFERENCES}

1. Sutherland, I. E., "A head-mounted three-dimensional display," Proceedings of fall joint computer conference, part I (AFIPS '68 (Fall, part I)), 757-764 (1968).

2. J.P. Rolland and H. Hua, "Head-mounted display systems," Encyclopedia of Optical Engineering, Ronald G. Driggers, Craig Hoffman, Ronald Driggers, Taylor \&amp; Francis (2003).

3. Roland Winston, Juan C. Miñano and Pablo Benítez, [Nonimaging Optics], Academic Press, Chapter 9 (2005).

4. José M. Infante Herrero, "Optical Systems Design using SMS method and optimizations," Ph.D. Thesis, Universidad Politécnica de Madrid (2013).

5. G.D. Wasserman and E. Wolf, "On the theory of aplanatic aspheric systems," Proc. Phys. Soc. B 62, 2-8 (1949).

6. D. Lynden-Bell, "Exact optics: a unification of optical telescope design," Mon. Not. R. Astron. Soc. (334), 787-796 (2002).

7. R. V. Willstrop and D. Lynden-Bell, "Exact optics - II. Exploration of designs on- and off-axis," Mon. Not. R. Astron. Soc. (342), 33-49 (2003).

8. G.W. Forbes, "Shape specification for axially symmetric optical surfaces," Opt. Express (15), 5218-5226 (2007).

9. G.W. Forbes, "Robust, efficient computational methods for axially symmetric optical aspheres," Opt. Express (18), 19700-19712 (2010).

10. Jiayao Liu, Juan C. Miñano, Pablo Benítez, Lin Wang, "Single optical surface imaging designs with unconstrained object to image mapping," Proc. SPIE 8550, Optical Systems Design 2012, 855011 (December 18, 2012).

11. Jiayao Liu, Juan C. Miñano, Pablo Benítez, "Single optical surface imaging designs with unconstrained object to image mapping with non-rotational symmetry," Proc. SPIE 9131, Optical Modelling and Design III, 91310B (May 1, 2014).

12. Orestes N. Stavroudis, [The Mathematics of Geometrical and Physical Optics: The k-function and its Ramifications], Wiley-VCH, Berlin, 97-114 (2006).

13. Dirk J. Struik, [Lectures on Classical Differential Geometry: Second Edition (Dover Books on Mathematics)], Dover Publications (1988). 